\title{
Le double jeu de la Baba Yaga : effets psychiques et socioculturels d'une performance théâtrale contemporaine
}

Sibylle Lesourd

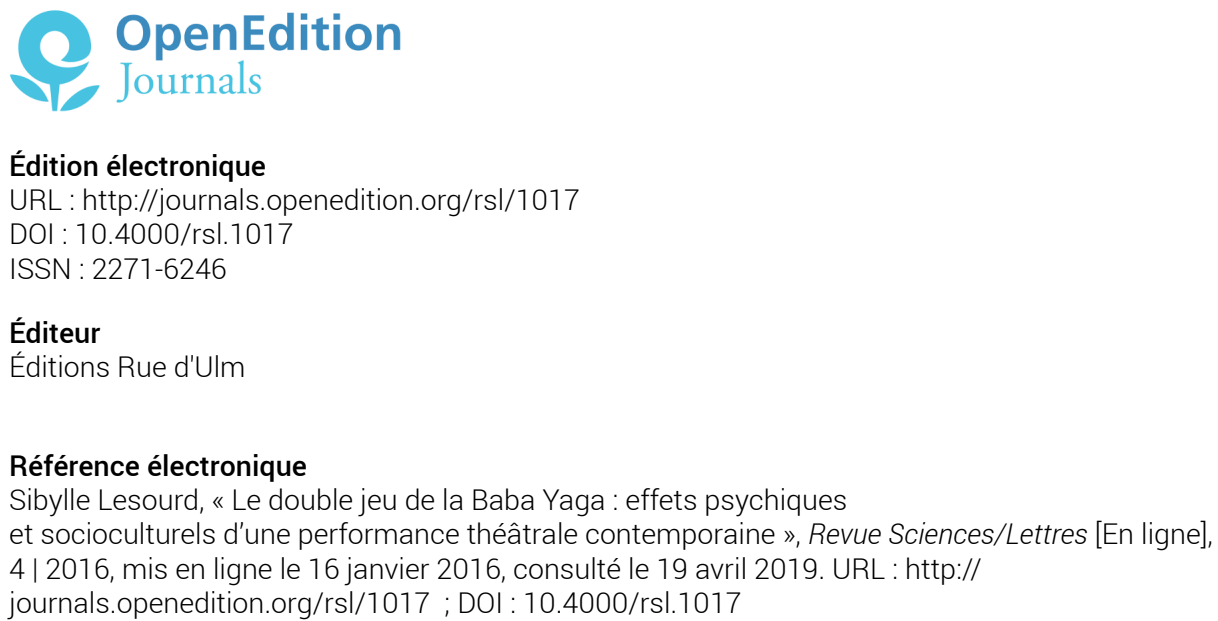

Ce document a été généré automatiquement le 19 avril 2019

(c) Revue Sciences/Lettres 


\section{Le double jeu de la Baba Yaga : effets psychiques et socioculturels d'une performance
théâtrale contemporaine}

Sibylle Lesourd

En 1994, la compagnie des Briciole de Parme, une troupe majeure du théâtre italien pour le jeune public, a créé le spectacle La Poupée dans la poche d'après le conte russe Vassilissa la Belle. Or le désir de communication avec l'enfant spectateur, au cœur de la recherche artistique des Briciole, a conduit à faire du conte le lieu d'une expérience active : la Baba Yaga, incarnée par une comédienne seule en scène, choisit une enfant parmi le public pour qu'elle joue le rôle de Vassilissa. Ce phénomène, dépassant largement le cadre de la participation traditionnelle, est une véritable capture scénique. En faisant éprouver aux jeunes spectateurs l'ambivalence de la Yaga, la comédienne va permettre à l'enfant capturée de vivre sous nos yeux une expérience initiatique.

2 La dramaturgie du spectacle repose tout entière sur la technique théatrale de la comédienne, exigeante et atypique, dans la mesure où elle comporte une dimension aléatoire et néanmoins maîtrisée. Flavia Armenzoni, créatrice du rôle de la Baba Yaga, l'a joué plusieurs centaines de fois puisque La Poupée dans la poche voyage depuis vingt ans à travers l'Europe et le monde. Cette artiste pionnière du théâtre pour l'enfance nous a fait part de son expérience en novembre 2011, au Teatro al Parco de Parme. 
Illustration 1 - La Poupée dans la poche (affiche du spectacle).

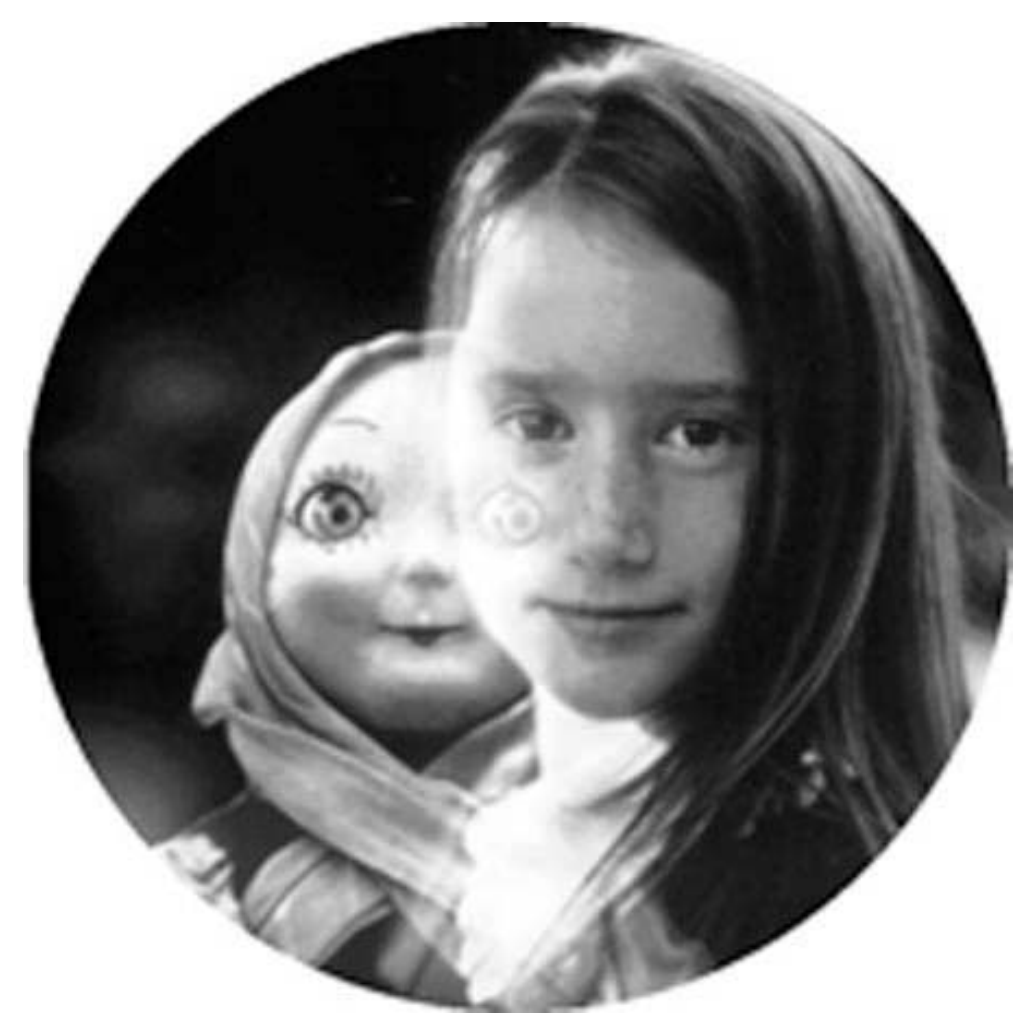

\section{Entretien avec Flavia Armenzoni}

\section{Comment est née l'idée de ce spectacle?}

À cette époque, j'avais envie de monter une "petite forme ${ }^{1}$ » et de la jouer seulement dans les environs, sans envisager de tournée à l'étranger. En effet je revenais tout juste d'une tournée très éprouvante en France où j'avais emmené mon enfant tout petit. Avec la metteuse en scène Letizia Quintavalla, nous avons eu l'idée d'aller dans les écoles maternelles pour nous adresser aux très jeunes enfants. Nous voulions travailler sur le petit, dans une recherche de simplicité absolue. En ce qui concerne le contenu du spectacle, nous recherchions une matière narrative car depuis quelques années la compagnie des Briciole avait pris l'habitude de monter des spectacles de narration ${ }^{2}$ qui avaient beaucoup de succès. Un jour, Letizia a découvert un livre qui faisait beaucoup parler de lui en Italie : Femmes qui courent avec les loups, de Clarissa Pinkola Estés ${ }^{3}$. C'est un livre de psychologie très simple, qui ne recevrait pas une grande considération des puristes, mais qui à nos yeux est de grande valeur. L'auteure y reprend les contes dans leur structure archaïque ; ce sont des contes qui concernent les femmes. Ainsi l'histoire de Vassilissa que je raconte n'est pas celle d'Afanassiev, elle est différente : par exemple il n'y a pas la fin heureuse, Vassilissa ne se marie pas. À l'époque d'Afanassiev, les contes devaient nécessairement finir bien, avec le mariage et les enfants. Nous avons préféré prendre l'histoire du livre de Pinkola Estés, sans nous priver de confronter les deux versions.

5 D'où est venue l'idée de faire intervenir une enfant du public? 
Cette idée est née un peu par hasard, ou plutôt par nécessité. Vois-tu, nous étions deux à travailler sur le spectacle, Letizia Quintavalla et moi. Or la protagoniste du conte était une enfant. Et nous détestons mettre en scène des protagonistes enfants. Les adultes qui jouent des enfants... ce n'est pas que nous ne l'ayons jamais fait, mais... c'est toujours quelque chose d'un peu étrange. Il nous fallait donc raconter l'histoire d'une protagoniste que nous ne pouvions pas incarner nous-mêmes : elle pouvait être prise dans le public ! Et du coup, la fonction de mon personnage serait de l'inciter à l'action. C'est sur cette base qu'on a commencé à travailler. Le plus difficile était qu'il me fallait répéter sans la protagoniste de l'histoire! Parce que quand une enfant a joué une fois avec moi, elle ne peut plus le refaire, puisqu'il faut nécessairement qu'elle découvre les choses au fur et à mesure. Alors il fallait à chaque fois répéter avec quelqu'un de différent.

Vous n'avez jamais pensé à faire intervenir une enfant comédienne?

Non, jamais. L'idée fondamentale était que l'enfant est la Vie, et que moi je suis le Théâtre. Si on fait intervenir une enfant comédienne, on perd cette idée. Nous nous appuyions à la fois sur ce présupposé théorique et sur notre connaissance des enfants. Nous savons bien qu'un enfant qui joue la comédie peut être la chose la moins théâtrale qui soit. Au contraire nous voulions utiliser la spontanéité, la surprise, la vie au plein sens du terme.

Quelles ont été les étapes de création du spectacle?

En fait nous avons commencé à jouer presque tout de suite, après un très petit nombre de répétitions. Il n'y avait pas encore de texte, j'étais dans l'improvisation. J'avais seulement la poupée, dont je ne savais pas encore très bien quoi faire. Avant d'arriver à savoir comment mener ce spectacle, nous avons fait de nombreuses tentatives, même erronées. Une fois on est allées dans une école et j'ai joué la sorcière d'une façon horriblement méchante; ensuite, je changeais de ton et je faisais la poupée ${ }^{4}$. La petite fille ne comprenait plus rien du tout. Elle n'était pas en mesure de comprendre un degré de fiction aussi avancé, surtout dans une école où il n'y avait aucun dispositif de son, de lumière... La pauvre enfant, je pense que je l'ai traumatisée pour toute sa vie. Maintenant, les choses sont pensées dans les moindres détails. Les lumières ne doivent être ni trop faibles, ni trop fortes. Il y a un jeu sur trois couleurs - le blanc, le rouge, le noir - et il faut que ce soit toujours ces couleurs-là. La taille des objets est aussi très étudiée. Tout cela est essentiel pour pouvoir créer une fiction qui ne fasse pas trop peur mais qui ne donne pas non plus trop confiance. C'est un savant équilibre qui doit être maintenu pendant toute la représentation.

11 Comment es-tu parvenue, peu à peu, à maîtriser ce spectacle?

12 Je dois dire que je n'avais pas du tout conscience de l'importance que prendrait ce spectacle. Au tout début, on était beaucoup plus proches de l'animation que du théâtre : on recherchait surtout un rapport direct avec les enfants. Mais aujourd'hui, ce n'est plus de l'animation, c'est vraiment du théâtre: le public - parmi lequel se trouve la protagoniste - est emporté dans une fiction théâtrale très forte. Et puis maintenant nous sommes très pointilleux sur le nombre de spectateurs, et nous écrivons une lettre aux enseignants pour expliquer qu'il faut au moins une classe d'enfants de cinq ans (parmi lesquels on choisit Vassilissa), il y a tout un règlement pour accueillir le spectacle. Parce que s'il n'y a que des enfants de trois ans, il est impossible de jouer! Nous sommes devenus terribles sur le plan contractuel. Au début on ne prenait pas toutes ces précautions. Elles sont le fruit de toutes mes expériences... 
Quand le brouillard s'est estompé dans ma tête, j'ai compris que le plus difficile serait de comprendre l'équilibre de la Baba Yaga. En effet, si elle est trop méchante, cela ne marche pas. C'est pour cela que doit s'établir un rapport subtil entre elle et la poupée : quand la figure du Bien apparaît, il est nécessaire que la figure du Mal disparaisse complètement. Mais si elle est trop gentille, cela crée d'autres difficultés. Pendant une certaine période, on a tenté une chose : je devais faire le clown, avec des chutes et des drôleries diverses. Mais comme cela faisait rire, cela ne faisait plus peur. Alors ça n'allait pas. Le danger d'un tel spectacle, c'est que le public peut décider de te manger. Il faut gérer le public, parce qu'il faut que ce soit du théâtre, que ce soit sacré, d'une certaine façon. Quand le public est si proche de toi, en plus, ça fonctionne un peu comme le squash : la balle te revient avec la même intensité que celle que tu as mise dans la frappe. Si tu parles trop fort, ils parlent trop fort. Je ne devais pas être seulement actrice, mais aussi éducatrice d'un comportement et d'un rituel.

15 J'ai compris peu à peu que pour être bonne, il faut se faire oublier. Toute ta concentration doit être mobilisée pour guider la petite fille, pour lui faire vivre cette expérience. Si ton regard abandonne la petite fille, elle ne devient plus rien. Ça, c'est une règle théâtrale : la petite fille n'est pas une actrice. Un acteur est capable de maintenir l'attention même s'il n'est pas protagoniste; une petite fille, non. C'est pour cela que tous les mouvements de Vassilissa sont chorégraphiques, dans le spectacle. Il fallait trouver une astuce pour la faire évoluer en permanence devant le public.

Illustration 2 - La pantomime de Vassilissa.

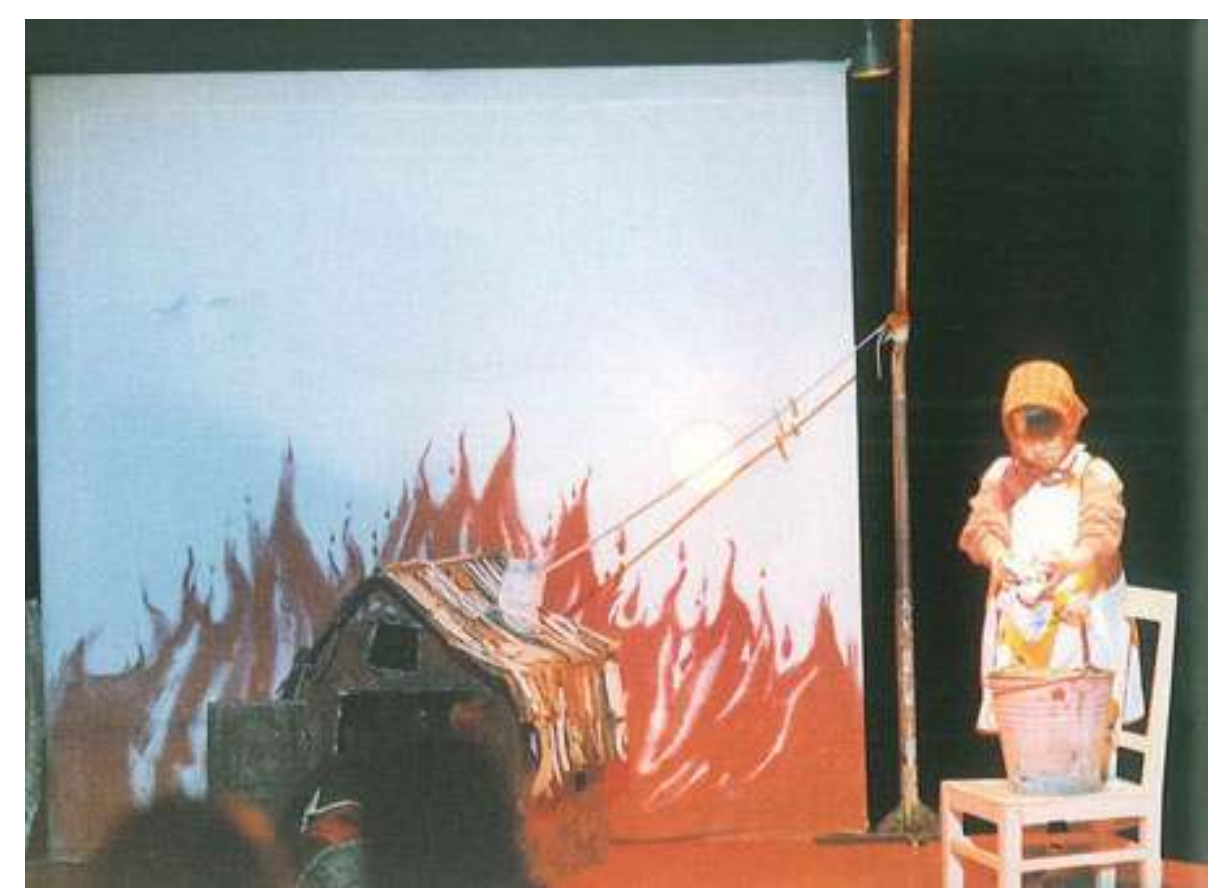

Pour pouvoir la maintenir active, théâtrale. Pour qu'elle puisse inventer et pour que le public puisse s'apercevoir que c'est elle qui invente. J'ai été impitoyable avec Laura ${ }^{5}$, l'actrice qui m'a remplacée pour faire le spectacle. Quand elle faisait la danse du feu, elle se laissait aller, les yeux fermés. Je lui ai dit: non! Ce n'est pas comme cela qu'il faut le 
faire, parce que si tu fermes les yeux, la petite disparaît. Si au contraire tu fixes intensément la petite fille, alors il peut arriver qu'elle danse avec toi. Tu dois être une complice. Évidemment, les actrices ont la tentation de jouer. Alors que tu es meilleure si tu ne joues pas trop : tu es actrice, éducatrice et aussi metteuse en scène de ce qui arrive.

Comment choisis-tu, à chaque fois, la fillette qui va jouer Vassilissa?

L'expérience que nous avons faite au début a été de demander aux institutrices. Mais après quelques représentations, j'ai vu que ce procédé ne marchait pas bien. Mon instinct m'aurait poussé vers d'autres petites filles. J'ai vite compris que les maîtresses indiquaient toujours les premières de la classe, alors que ce ne sont pas forcément celles qui ont besoin de cette expérience, qui ont quelque chose à l'intérieur qu'elles ne parviennent pas à faire sortir... Et donc ce ne sont pas forcément celles qui se prêtent au jeu de la façon la plus émouvante. J'ai donc changé de méthode. Je me suis dit qu'après tout c'était un spectacle fondé sur l'intuition féminine; c'est en tout cas ce que dit l'auteure du livre qui nous a inspirées. Alors je me suis dit: je dois me fier à ma poupée dans la poche, me laisser guider par ma propre intuition. Et à partir de là, j'ai eu des expériences grandioses. En fait, toute la première partie du spectacle est prévue pour comprendre qui, dans le public, peut être Vassilissa. Ce n'est pas nécessairement la moins timide, la plus exubérante. Peut-être justement ce sera la plus timide de toutes. L'intuition peut naître d'un geste, d'une question, d'un regard.

9 Nous entrons ici dans la première phase du spectacle... En guise de préambule, tu poses aux enfants rassemblés toute une série de questions.

Oui, cette première phase est conçue pour observer les enfants. D'abord je leur demande un mouchoir. Il faut que je fasse croire à un besoin véritable. C'est une façon de leur dire : j'ai besoin de vous, je suis faible. Les questions, elles, permettent d'étudier le public ; mais elles sont aussi prévues pour faire comprendre aux enfants que ce sont eux, les protagonistes ; et que ce qu'ils font, ce qu'ils disent, est bien, et qu'il n'y a aucun jugement porté sur eux. Il faut entrer dans une relation complètement à égalité. Et puis je ne pose pas des questions normales, celles qu'on poserait habituellement à un enfant de quatre ans. Ce sont même des questions difficiles, philosophiques ${ }^{6}$, auxquelles ils peuvent répondre de manière créative. Dans cette première partie, au moindre sourire de la part d'un spectateur adulte, il faut réagir. La plupart du temps il suffit d'un regard et l'adulte, s'il est intelligent, se souvient de nos consignes ${ }^{7}$ et les respecte parce qu'il comprend quel projet il y a derrière.

\section{Pourquoi ensuite racontes-tu en entier l'histoire de Vassilissa?}

Parce que si tu sais comment ça va finir, peut-être que tu vas oser monter sur le tapis rouge. Mais si tu te demandes si la sorcière va te manger - et les enfants croient vraiment qu'ils peuvent être mangés - tu n'y vas pas. Donc nous avons fait un travail de résumé pour ne garder que les éléments principaux du conte, en sacrifiant même de beaux passages. L'idée essentielle était de créer un langage commun. Un seul geste doit pouvoir te rappeler un moment précis du conte. C'est pourquoi je raconte l'histoire trois fois. La première fois avec beaucoup de mots, et en associant des gestes à certains mots. La deuxième fois il y a moins de mots et je m'appuie sur la gestuelle, en m'assurant que le public a compris les parties essentielles. Et la troisième fois, il ne reste que les gestes. En particulier, le geste fondamental. (Elle mime ce geste qui consiste à nourrir la poupée, de la bouche à la bouche. Un geste primordial et fascinant.) 

quand j'ai créé le spectacle car j'avais un enfant du même âge) et je lui parle tout bas comme si j'étais sa maman. Le public ne m'entend pas. Je lui dis: «Elle te plaît, ma poupée? Tu sais qu'elle est magique? Mais elle est magique pour de vrai. Elle t'aidera toujours. » Et puis encore : «Faisons un jeu. Moi je vais me déguiser en sorcière. » Je lui dis de m'attendre et je sors. Ces mots sont très importants parce qu'ensuite elle doit s'y fier complètement. À ce moment il peut toujours arriver qu'une enfant refuse. Alors la règle est de ne pas insister - il faut seulement bien s'assurer qu'elle ne veut vraiment pas le faire. Alors, tout ce que je fais doit être théâtral. Si une petite fille pleure, ou si elle fait pipi (car tout cela arrive !), je redeviens narratrice, j'enlève mes éléments de costume et je dis: "Cette Vassilissa est très fatiguée et retourne à la maison. Arrive une autre Vassilissa. » On peut changer de petite fille à tout moment du spectacle. Mais il y a des moments où c'est vraiment rude, techniquement. Par exemple, lorsqu'arrive la Baba Yaga. C'est un moment de peur : pour la première fois, on baisse la lumière ; j'entre en scène avec la maison qui tourne sur la tête...

Illustration 3 - Baba Yaga, avec louche et couvercle.

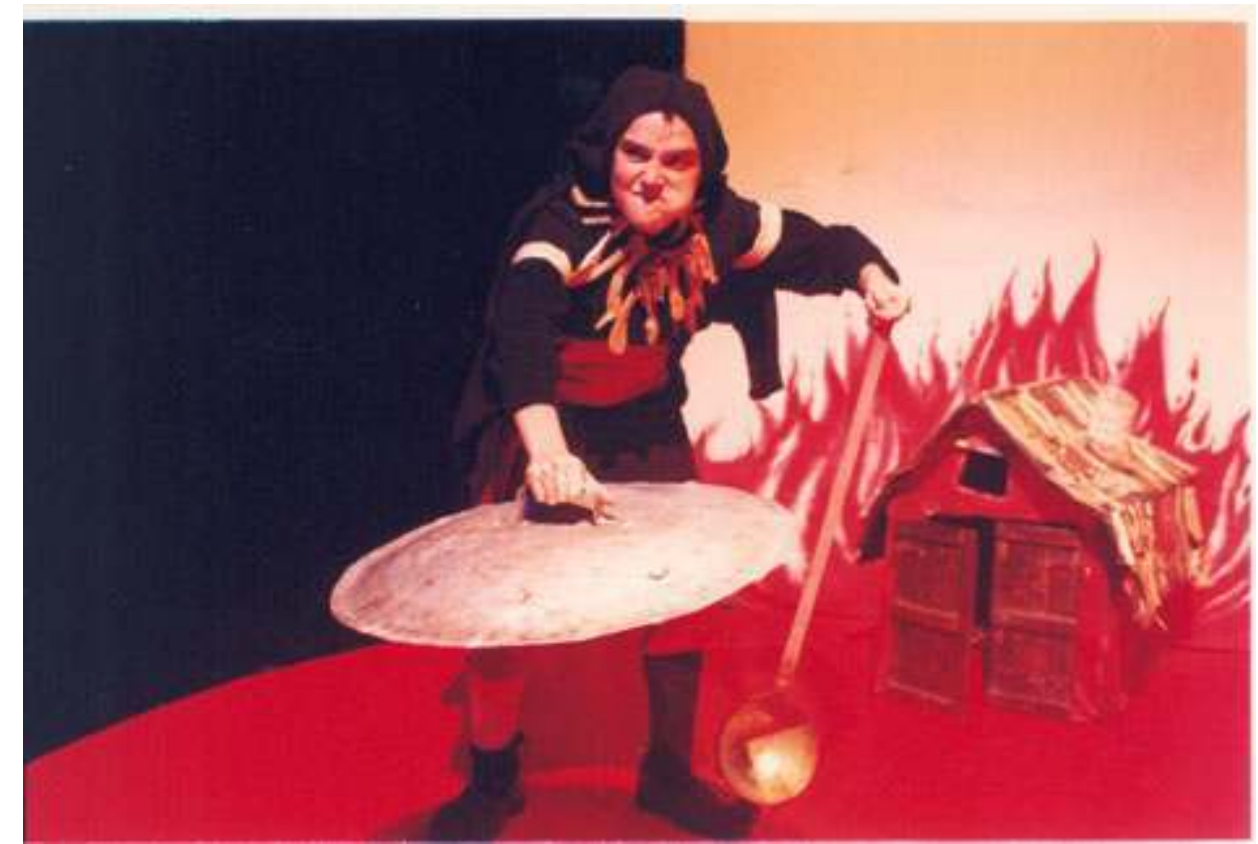

27

Dans certains cas, j'ai vu l'enfant s'enfuir à ce moment-là! Tu es effrayante avec ton visage déformé... Il est difficile de reconnaître en toi la conteuse ou la maman. 

tu peux la faire inventer. Il y a des jours où les petites filles imaginent des tas de choses, des façons étonnantes de faire la cuisine... et d'autres jours où elles ne trouvent rien parce qu'elles ont peur. Le public, quand il voit ce spectacle, est toujours conquis, mais nous actrices savons bien que certaines représentations sont de qualité bien supérieure. Cela dépend de la qualité du rapport entre réalité et fiction. La petite doit comprendre que tu fais la méchante pour son bien. Que plus les travaux sont difficiles, plus elle se montre vaillante. Que plus ils sont impossibles, plus tu l'aideras. Ce qui est merveilleux, c'est le degré de complicité d'actrices qui se met à exister entre une petite fille qui n'a pas conscience de ce qu'elle fait, et toi qui as étudié aussi intensément cette chose que vous faites ensemble. J'adore quand elles me mentent et me cachent l'existence de la poupée, parce que cela montre qu'elles ont compris toute la fiction... ou qu'elles pensent que la poupée est vraiment magique.

Illustration 4 - La sorcière cachée.

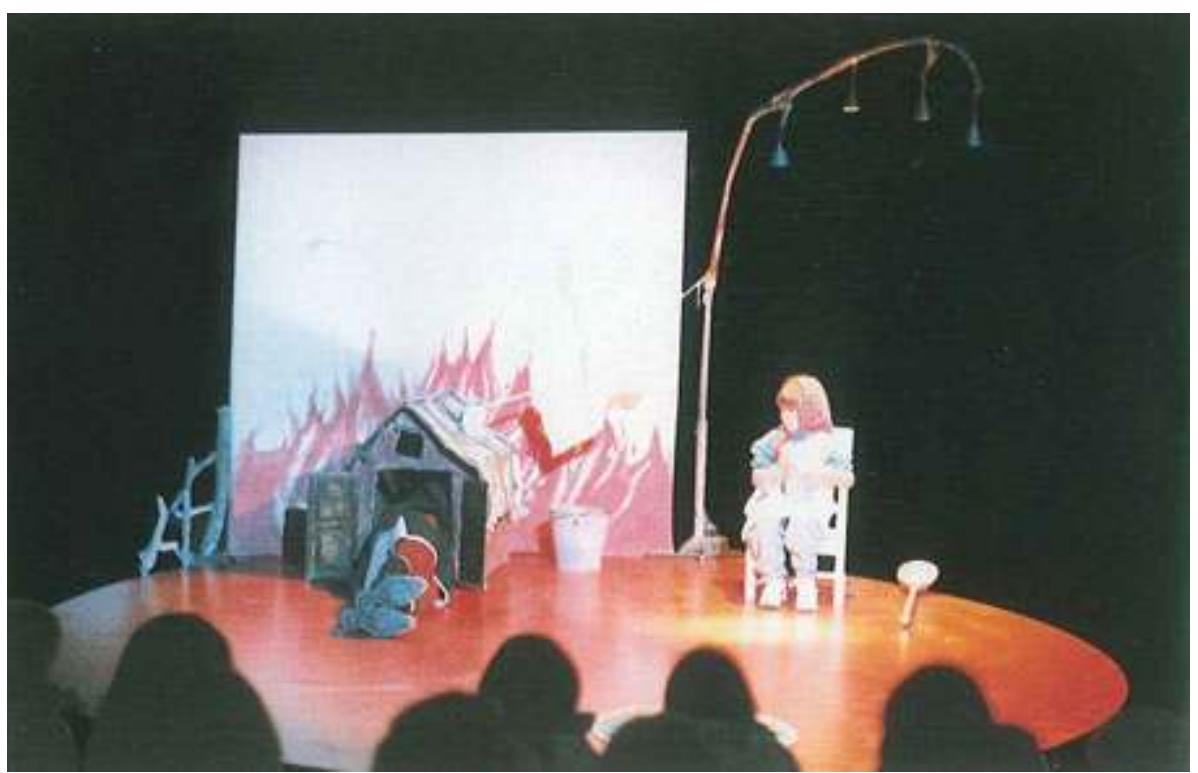


Finalement tu as emmené ce spectacle en tournée en Europe et dans le monde. As-tu constaté des différences culturelles dans la réception du spectacle?

Pour jouer ce spectacle, en Italie et ailleurs, il est important d'être à l'écoute du lieu où tu vis. Chaque groupe est différent, il faut jouer d'une façon différente pour chaque public. Lorsque tu as beaucoup d'expérience, tu te mets à avoir des préjugés, à te dire : ce type de public-là, je l'ai déjà vu. Quand j'étais à Paris, par exemple, j'ai vite remarqué que jouer à Chaillot, ce n'est pas comme jouer à Saint-Denis. Les enfants de Chaillot lavaient beaucoup moins bien les culottes ! Déjà, rien que dans leur façon de les tenir... Alors qu'à Naples! (Elle mime avec expressivité, et tour à tour, les réactions dégoûtées des enfants de Chaillot et l'enthousiasme des gamines de Naples qui prenaient les culottes à pleines mains pour les plonger dans l'eau.) Ce n'est pas une critique, bien sûr. Mais ce spectacle met en scène le social. Avec Letizia, on avait cherché des tâches faciles à réaliser pour une enfant de quatre ans. Moi j'appartiens à une génération où la petite fille aidait la maman, et une des premières choses qu'on apprenait à faire, c'était laver à la main. Peut-être pas les culottes, d'accord, et c'est vrai que ça peut sembler répugnant de laver cet accessoire intime de la Baba Yaga. Mais par exemple, étendre le linge me semblait quelque chose de très évident. Maintenant, il arrive que les enfants ne sachent plus du tout comment faire ! Au Canada, par exemple, c'était impossible. On aurait dit que les enfants n'avaient jamais vu un séchoir. Bien sûr, chez eux, tout le monde a le sèche-linge automatique! Et pourtant ce geste culturel était important pour le spectacle.

Était-il difficile de jouer ce spectacle dans une langue étrangère que tu maîtrisais imparfaitement, comme le français?

J'avais déjà joué quelques spectacles en français mais il s'agissait simplement d'apprendre un texte. Avec La Poupée dans la poche, c'était autre chose : il me fallait comprendre ce que disait la petite fille, être capable d'improviser par rapport à ses répliques... Le plus difficile était de jouer dans les quartiers de forte immigration. Les enfants, en effet, y apprennent la langue au cours de leur scolarité. Les enseignants me disaient qu'ils connaissaient le français mais en vérité ils savaient peut-être dire "un cahier », ils connaissaient le français de la vie scolaire, mais pas le français du quotidien. Du coup, ils ne comprenaient pas l'histoire. Pour me faire comprendre, j'ai dû parler toutes les langues ; parfois j'aimais autant faire des gestes et revenir à l'italien. J'ai eu toutefois une très belle expérience à Mulhouse : j'avais face à moi des enfants en majorité africains, je ne savais pas de qui je pourrais me faire comprendre. J'ai choisi une petite chinoise de quatre ans, mais mal m'en a pris car elle ne comprenait pas du tout cette actrice italienne qui parlait français avec un accent... Par chance, les autres enfants, eux, me comprenaient très bien! Alors ils se sont mis à guider la petite Vassilissa par gestes, en se cachant de la sorcière ! Comme c'était beau, ce jour-là. Cette complicité de tous les enfants a permis à la petite, bien que ne comprenant pas la langue, de triompher de la Baba Yaga. Pour sa plus grande joie! 


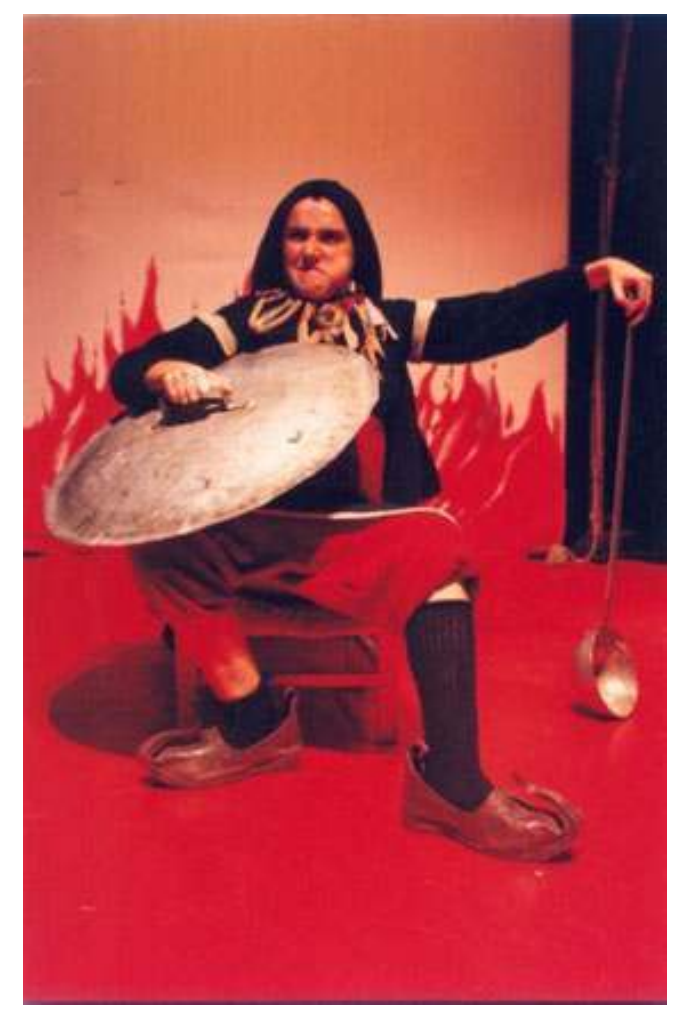

\section{Analyse}

37 Dans cette performance théâtrale, l'expérience vécue par l'enfant capturée est la rencontre avec la figure mythique de la Baba Yaga. Celle-ci est ressaisie par la psychanalyse : pour Clarissa Pinkola Estés, dans Vassilissa la Belle, la Baba Yaga incarne la «mère sauvage » avec qui l'enfant doit trouver une affinité pour nouer un rapport plus intuitif à la vie, en se séparant progressivement de l'archétype de la mère douce et bonne. Pour que ce trajet initiatique puisse se réaliser, la comédienne met en place un double jeu où, sous l'apparence du manichéisme, la figure maternelle laisse entrevoir son ambivalence et aide ainsi l'enfant à grandir.

Ce double jeu ne peut s'établir immédiatement et requiert un temps de préparation. C'est pourquoi dans la première partie du spectacle, la comédienne ne joue pas et raconte aux enfants l'histoire de Vassilissa la Belle. Ce faisant, elle met en place les grands repères structurants du conte en les ritualisant progressivement. Face à la méchante sorcière, la ressource essentielle de l'héroïne est la poupée que lui a confiée sa mère avant de mourir et qui lui porte conseil dès qu'elle la nourrit. Le geste symbolique exprimant l'acte de nourrir la poupée est riche pour l'interprétation psychanalytique: la main va de la bouche de l'enfant à celle supposée de la poupée, installée dans une poche au niveau de son ventre. L'accomplissement de ce geste « de la bouche à la bouche » met l'accent sur l'oralité et traduit le rapport fusionnel avec la mère nourricière, qui s'oppose bien sûr à la mère dévoratrice, la Baba Yaga. 

un rapport maternel : l'ayant entraînée sur la scène et dotée des accessoires de l'héroïne du conte (un capuchon, la poupée dans la poche), elle la prend sur ses genoux et la berce,

Illustration 6 - L'enfant sur les genoux.

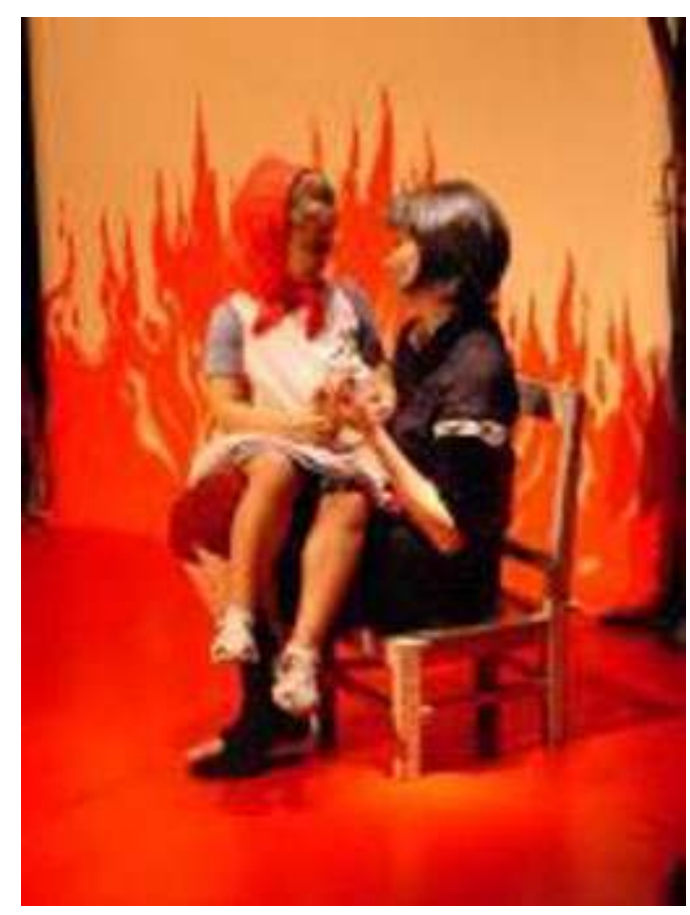

lui murmurant des choses dans le creux de l'oreille. À ce stade du spectacle, elle doit apparaitre aux yeux de l'enfant capturée - et à ceux des jeunes spectateurs - sous les traits d'une figure maternelle incarnant la douceur et la bonté. Au moment de l'entrée terrible de la Baba Yaga et de son isba, il est essentiel que l'enfant éprouve de la peur. Certes, il ne faut pas qu'elle ait trop peur, sinon elle sera incapable de faire quoi que ce soit. Mais il s'agit de susciter une peur suffisante pour que la dimension initiatique opère et pour que le jeune public entre sérieusement dans la fiction théâtrale. D'où l'importance de susciter une première apparition impressionnante, avec d'abord la maison tournante sur la tête de la sorcière, puis la découverte du visage métamorphosé de la comédienne (un œuf déformant sa joue), soudain agressive dans son adresse à l'enfant. Celle-ci, très jeune, peut hésiter à reconnaître la figure maternelle complice qui vient de la quitter. $\mathrm{Si}$ l'enfant reste face à la sorcière (ce qui est déjà en soi une démonstration de courage), le double jeu va pouvoir se mettre en place.

Pour la comédienne, il s'agit d'incarner à la fois l'archétype de la Méchante (celle qui donne à l'enfant des tâches difficiles, voire impossibles à réaliser et menace de la dévorer en cas d'échec) et la figure aidante et gentille, celle de la Poupée qui fait en sorte que l'enfant parvienne à réaliser les tâches en question. Or face à ce personnage ambigu et contradictoire, l'enfant risque de ne pas trouver sa place dans le spectacle. La solution théâtrale est que les deux figures doivent s'exclure radicalement l'une l'autre pour établir la dichotomie avec clarté. Il y aura d'une part la sorcière infligeant les épreuves, debout face à l'enfant ; lorsqu'elle se retire, c'est une voix off, extrêmement douce, qui prend en charge le discours suggestif et tendre de la Poupée - «Regarde à tel endroit, essaie de faire tel geste, c'est bien mon trésor, c'est bien mon cœur. » Ainsi, lorsque l'enfant 
exécute l'épreuve, elle est seule sur le plateau. Cachée en fond de scène, la comédienne ne perd pas de vue sa pantomime et encourage son initiative, la guide quand c'est nécessaire, pallie les éventuelles difficultés, adapte enfin l'exigence en fonction de ce qu'elle se révèle capable de réaliser.

Dans le conte populaire, l'expérience initiatique de Vassilissa passe par la réalisation de tâches ménagères - faire la lessive, accrocher le linge, plier un drap, balayer le sol - qui lui permettent, symboliquement, de sortir de l'enfance et de devenir une femme, initiée par une figure maternelle oppressante. La transposition théâtrale du conte, qui en fait une expérience active, soumet également l'enfant capturée à une contrainte forte : en effet elle ne peut pas se soustraire à la dramaturgie préétablie, qui lui a été répétée maintes fois, tant et si bien qu'elle doit y jouer son rôle. Mais elle est libre d'inventer la façon dont elle s'acquittera de chacune des épreuves - comment elle nettoiera les effets personnels de la Baba Yaga, comment elle séparera les grains de riz blancs et noirs... Le rôle de la Poupée est de l'aider à trouver en elle-même des ressources qu'elle ignorait posséder jusqu'alors.

Dans ses représentations archaïques, la Yaga incarne le passage entre le monde des vivants et le monde des morts. La réappropriation de cette figure mythique par les Briciole vise plutôt à réaliser sur la scène une rencontre authentique entre la Vie et le Théâtre. La Yaga permet le passage d'une dimension à l'autre : en initiant l'enfant à la fiction théâtrale, elle lui indique un chemin pour grandir. L'effet quasi thérapeutique de ce spectacle, qui dans certains cas évoque le psychodrame ${ }^{8}$, relève en tout cas d'une catharsis ${ }^{9}$ de l'acteur. En effet, dans La Poupée dans la poche, les conflits latents autour de la figure maternelle peuvent être appréhendés symboliquement et surmontés réellement par l'enfant. En ce sens, l'expérience théâtrale est initiatique. Le double aspect apparemment irréconciliable de la mère nourricière et de la mère dévoratrice est perçu par l'enfant selon le schéma manichéen du conte, ce qui lui permet d'entrer en action, faisant confiance à l'une et résistant à l'autre. Mais ce faisant, elle prend conscience qu'en réalité il n'y a pas deux mères distinctes mais une seule figure maternelle ambiguë. L'ambivalence constitutive de la Baba Yaga s'inscrit progressivement dans son psychisme. En choisissant de jouer avec elle et en s'appuyant sur elle pour affirmer son identité propre, l'enfant est amenée à perdre ses illusions au sujet d'une mère qui serait toute bonté mais ne renonce pas pour autant à lui faire confiance et à voir en elle une alliée dans son chemin vers l'indépendance. À la fin de l'histoire, c'est bien la sorcière qui lui remet le feu et l'incite à brûler la maison de sa marâtre pour se rendre libre.

Les effets psychiques du spectacle ne concernent pas seulement l'enfant capturée. Le phénomène de l'identification, décrit par Aristote, fonctionne parfaitement dans la mesure où les jeunes spectateurs ont conscience que Vassilissa a été choisie parmi eux et qu'il aurait pu s'agir de n'importe qui parmi l'assistance. Dès lors, les longues pantomimes de Vassilissa, livrée à elle-même sur le plateau, sont suivies par une assistance fascinée qui retient son souffle. En vérité, la mise en valeur de l'enfant capturée repose beaucoup sur le jeu de la comédienne : celui-ci ne doit pas être trop appuyé afin qu'un équilibre se crée entre le Théâtre et la Vie. L'objectif est de rendre l'enfant aussi théâtrale que possible, et donc de la mettre en action, car c'est en la voyant active et expressive que le public sera saisi de vertige et partagera pleinement l'expérience initiatique.

Au-delà de son impact psychique, le spectacle La Poupée dans la poche révèle souvent les problématiques sociales et culturelles des groupes auxquels il se confronte. Dès l'instant où un membre du public a l'opportunité d'agir sur scène et d'y imposer sa propre 
gestuelle, le théâtre met facilement en scène les réalités sociales. De plus, il est arrivé parfois que le spectacle apporte, même sans le rechercher consciemment, une réponse à une problématique culturelle locale vis-à-vis de laquelle la Baba Yaga a pu faire figure de catalyseur. C'est ce que révèle l'anecdote de la petite Chinoise de Mulhouse : la solidarité inattendue de tous les jeunes spectateurs, ligués contre une figure oppressante, a permis au spectacle de se tenir mais surtout a contribué fortement à l'intégration d'une fillette d'une autre culture au sein de la communauté enfantine. intéressante à la fois par ses effets psychiques et socioculturels. Mais que reste-t-il, en définitive, de la figure mythique des contes populaires dans cette performance théâtrale ? Esquissons quelques hypothèses pour finir :

- À un premier niveau, la Baba Yaga est l'archétype du Mal absolu. Elle semble alors se confondre avec la sorcière des contes occidentaux, celle qui fait obstacle au héros. Pour incarner ce personnage redoutable, la comédienne doit trouver un équilibre dans son jeu afin de ne pas déclencher l'inhibition de l'enfant et d'être au contraire instrument de révélation.

- À un deuxième niveau, la Baba Yaga est une figure maternelle complexe et ambiguë. Grâce au double jeu de la comédienne, la performance théâtrale rend justice à l'interprétation psychanalytique de Pinkola Estés et prépare subtilement l'enfant à faire la transition de la mère douce à la mère sauvage.

- À un troisième niveau, enfin, la Yaga est une figure liminaire : elle incite à franchir une frontière symbolique, celle qui existe entre la Vie et le Théâtre, ou celle qui sépare certains groupes humains. Elle pousse ainsi à retrouver des communautés instinctives, primaires, endeçà des clivages culturels.

\section{NOTES}

1. Un spectacle de courte durée, avec peu d'éléments de décor.

2. La narration théâtrale est un genre apparu en Italie dans les années 1980, sous l'impulsion du créateur Marco Baliani. À l'origine, elle se caractérise par l'absence de décor et la présence en scène d'un narrateur-acteur qui livre un récit à son public.

3. Clarissa Pinkola Estés est une psychanalyste jungienne. Dans son ouvrage Femmes qui courent avec les loups, paru en 1992, elle choisit des contes centrés sur des figures féminines et elle met au jour les processus d'individuation et de connaissance de soi dont ils sont porteurs. Ainsi, son analyse du conte Vassilissa la Belle est axée sur l'histoire de la transmission, de la mère à la fille, de l'intuition féminine. (Voir Clarissa Pinkola Estés, Femmes qui courent avec les loups, Paris, Grasset, 1996).

4. Dans Vassilissa la Belle, l'héroïne a été envoyée dans la forêt par sa belle-mère et ses bellessœurs pour y récupérer le feu auprès de la Baba Yaga. Elle est aidée par la poupée que lui a confiée sa vraie mère avant de mourir: lorsqu'elle la nourrit, la poupée lui donne de bons conseils.

5. Il s'agit de Laura Magni. 
6. La comédienne demande par exemple aux enfants: "C'est quoi le temps? C'est quoi la mémoire?» Les enfants répondent spontanément et toutes les réponses sont acceptées avec beaucoup de sérieux.

7. Avant l'entrée dans la salle, les adultes se voient remettre un petit papier qui leur demande de contenir leurs réactions pendant le spectacle afin de laisser les enfants le vivre intensément.

8. Il s'agit d'une forme de thérapie inventée par le psychiatre américain Jacob Levy Moreno. Ce dernier récuse la psychanalyse selon Freud: au lieu de demander au patient de raconter, il préfère lui suggérer de s'exprimer par des actions et des gestes pour montrer les événements traumatiques qu'il a vécus et s'en délivrer. Voir Anne Ancelin Schützenberger, Le Psychodrame, Paris, Éditions Payot et Rivages, 2008.

9. La catharsis est une notion aristotélicienne: elle vise l'épuration des passions par la représentation théâtrale. Dans son acception originelle, la catharsis se produit chez le spectateur.

\section{RÉSUMÉS}

Le désir de communication avec le jeune public, au cœur de la recherche artistique des Briciole, conduit cette compagnie italienne à faire du conte Vassilissa la Belle le lieu d'une expérience active : la Baba Yaga, incarnée par une comédienne seule en scène, choisit une petite fille parmi l'assistance pour qu'elle joue le rôle de Vassilissa. Ce phénomène, dépassant largement le cadre de la participation traditionnelle, est une véritable capture scénique : par son ambivalence, la Baba Yaga permet à l'enfant d'effectuer sous nos yeux un parcours initiatique.

The wish to communicate with young audiences, which is the central preoccupation of the Briciole (an Italian theatre company), leads them to turn the tale Vassilissa the Beautiful into an active experience: Baba Yaga, performed by an actress who is alone on stage, picks a little girl from the audience to play the part of Vassilissa. Widely exceeding traditional participation, this phenomenon can be described as a form of stage capture: Baba Yaga's ambivalent nature enables the child to undergo an initiation rite before our very eyes.

\section{INDEX}

Mots-clés : théâtre, jeunesse, Italie, psychanalyse

Keywords : theatre, youth, Italy, psychoanalysis

\section{AUTEUR}

\section{SIBYLLE LESOURD}

Sibylle Lesourd, normalienne et agrégée de lettres modernes, doctorante en littérature comparée à l'université de Paris-Sorbonne. Professeur de français, rédactrice et responsable de la rubrique théâtre dans la Revue des livres pour enfants. Thèse en cours : «L'enfant protagoniste : naissance, mouvances et paradoxes d'une figure clé du théâtre contemporain pour la jeunesse en France et en Italie des années 1960 à nos jours. » sibylle.lesourd@gmail.com 